\title{
Functional Differences between and across Different Regions of the Apical Branch of Hippocampal CA1 Dendrites with Respect to Long-Term Depression Induction and Synaptic Cross-Tagging
}

\author{
Suhel Parvez, Binu Ramachandran, and Julietta U. Frey \\ Department of Neurophysiology, Leibniz-Institute for Neurobiology, 39118 Magdeburg, Germany
}

\begin{abstract}
The hippocampus is an ideal system to study synaptic plasticity in the context of learning and memory. The induction, expression, and interaction of long-term potentiation (LTP) as well as long-term depression (LTD) are essential elements for the functioning of complex networks in information processing and storage. Here we investigated whether different loci at the apical dendritic branch of CA1 pyramidal neurons are characterized by different capabilities to induce, express, and interact with LTP and LTD in hippocampal slices in vitro. We found that high-frequency stimulation resulted in longer-lasting forms of LTP in proximal and distal parts of the apical dendrites, whereas low-frequency stimulation induced longer-lasting LTD in distal but not at proximal parts. Interestingly, processes of "synaptic cross-tagging" could be described for any form of LTP transformation from early-stage LTP (E-LTP) into late-phase LTP (L-LTP) in distal and proximal parts, but for LTD, only at the distal part but not for the proximal part, although low-frequency stimulation at the proximal input, which resulted here only in a short-term depression, was paradoxically able to reinforce E-LTP into L-LTP at distal parts. We have identified protein kinase Mzeta (PKMzeta) as the LTP-specific, synthesized plasticity-related protein transforming E-LTP into L-LTP by strong low-frequency stimulation in the apical CA dendrite by cross-tagging mechanisms.
\end{abstract}

\section{Introduction}

The hippocampal CA1 region is one of the major structures to study synaptic plasticity. In particular, most data obtained from that region were undertaken at apical dendrites of CA1 pyramidal neurons. Long-term potentiation (LTP; prolonged increase in synaptic efficacy after brief high-frequency trains of afferent fibers) as well as long-term depression (LTD; prolonged decrease in synaptic efficacy in response to low-frequency stimulation patterns to afferent fibers) can be induced in this region. We refer here only to NMDA receptordependent forms, which can express under distinct circumstances a protein synthesis-independent early-stage (E-LTD or E-LTP, $<4 \mathrm{~h}$ ) and a protein synthesis-dependent late phase (L-LTD or L-LTP, $>4 \mathrm{~h}$ ), although various forms of LTP and LTD exist (for review, see Massey and Bashir, 2007; Sajikumar et al., 2005a; Pöschel and Manahan-Vaughan, 2007; Frey and Frey, 2008). It is also well documented that long-lasting forms of LTP can be induced in several parts of the apical CA1 dendrite, regardless of whether it is studied at proximal or distal sites (Frey and Morris, 1997). However, less is known about forms of LTD expression at different parts of apical CA1 dendrites. The cellular mechanisms for the

Received Nov. 23, 2009; revised Feb. 3, 2010; accepted March 10, 2010.

This work was supported by grants from the Deutsche Forschungsgemeinschaft (1034-7) and Sonderforschungsbereich (779-B4) to J.U.F. and a fellowship of the Alexander-von-Humboldt Foundation to S.P. We are grateful to Diana Koch and Anja Apel for their excellent technical assistance.

Correspondence should be addressed to Prof. Dr. Julietta U. Frey, Department of Neurophysiology, LeibnizInstitute for Neurobiology, Brenneckestrasse 6, 39118 Magdeburg, Germany. E-mail: frey@ifn-magdeburg.de.

DOI:10.1523/JNEUROSCI.5808-09.2010

Copyright $\odot 2010$ the authors $\quad 0270-6474 / 10 / 305118-06 \$ 15.00 / 0$ induction and expression of LTD (as well as LTP) can vary depending on the stimulation protocol, brain subregion, and stage of development (Kemp et al., 2000; Malenka and Bear, 2004; Pöschel and Manahan-Vaughan, 2007). Prolonged low-frequency stimulation (LFS) is required to induce a prolonged protein synthesis-dependent L-LTD, which also requires NMDA receptor activation, postsynaptic $\mathrm{Ca}^{2+}$ influx, and activation of a serine/threonine phosphatase cascade (Mulkey et al., 1994; Manahan-Vaughan et al., 2000; Sajikumar and Frey, 2003; Sajikumar et al., 2005a). Furthermore, heterosynaptic LTD is an activity-dependent form of a persisting decrease in synaptic efficacy that occurs at inactive synapses when postsynaptic depolarization and firing are initiated via another afferent pathway (Barry et al., 1996) However, a prolonged maintenance of that form was not yet described. Transient, heterosynaptic LTD is different from the heterosynaptic requirements to induce L-LTD as described previously (Sajikumar and Frey, 2004).

Some laboratories reported a relative inconsistency with respect to LTD forms and its duration in CA1, which could be, for instance, due to the lack of optimal stimulation protocols (Malenka and Bear, 2004). Thus, the duration of postsynaptic $\mathrm{Ca}^{2+}$ elevation has been postulated as an essential factor for differential induction of LTP or LTD (Mizuno et al., 2001). We, however, suggest that the location within the dendritic branch is one of the major contributing factors for the efficacy of the inputs to express longer-lasting forms of LTD. Here, we investigated whether different regions of the apical CA1 dendrite express specific forms of LTD. Further, as we had previously shown that 


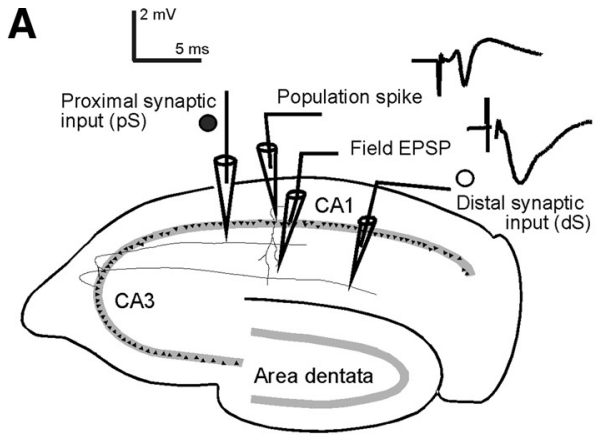

B

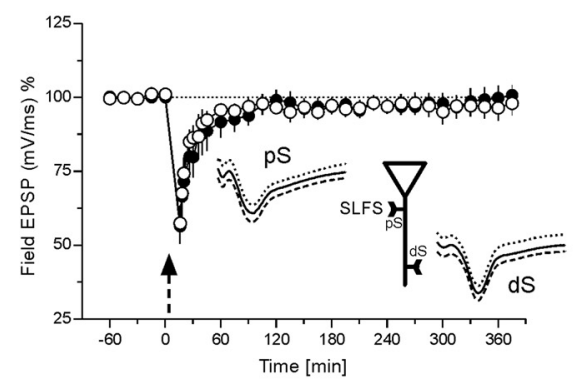

c

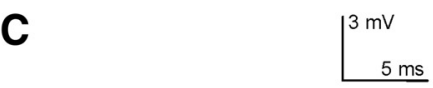

D

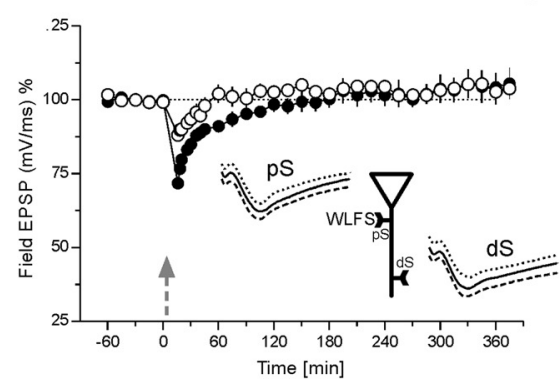

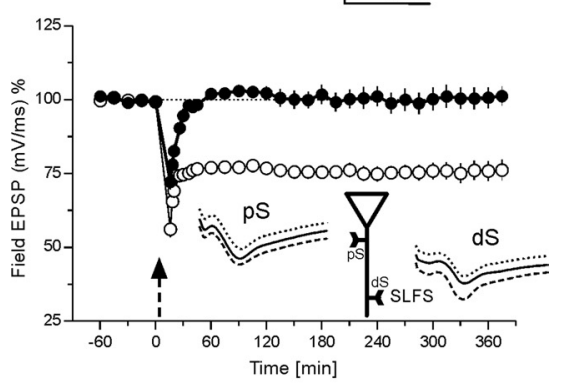

$E$

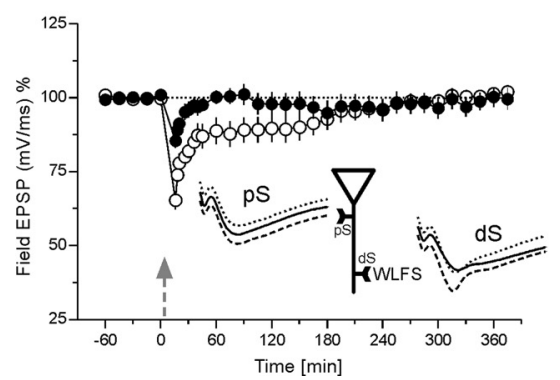

Figure 1. Induction and maintenance of different LTD forms depending on the region of its induction at apical CA1 neurons. The time course of field EPSP recordings are shown. $\boldsymbol{A}$, Schematic representation of a transversal rat hippocampal slice with electrode locations to stimulate two separate synaptic inputs $\mathrm{pS}$ (proximal) and $\mathrm{dS}$ (distal) in the stratum radiatum of the CA1 region is shown. Further, the locations of the recording electrodes for the population spike as well as the field EPSP from a single, stimulated neuronal population are provided. The analog traces are representative examples of recorded potentials at the two recording electrodes. $\boldsymbol{B}$, The time course of the slope of the field EPSP after SLFS within the proximal region of the apical dendrites $p S$ is presented (filled circles). Open circles represent the recordings from the distally located control synaptic pathway dS $(n=9)$. STD in the $\mathrm{pS}$ and a transient heterosynaptic depression in dS was seen. $C$, Application of SLFS to distally located inputs $d S$ are shown, which resulted in L-LTD (open circles). Filled circles represent recordings from the proximally located control synaptic pathway $\mathrm{p} S$ which expressed a heterosynaptic transient potentiation; $(n=20)$. $\boldsymbol{D}$, The time course of the slope of the field EPSP after WLFS to $p S$ is provided, which only expressed STD (filled circles). Open circles represent recordings from the control dS showing a transient heterosynaptic depression $(n=7)$. $E$, Application of WLFS to dS resulted in E-LTD (open circles). Filled circles represent recordings from the control synaptic pathway $p S$ in this series of experiments showing a heterosynaptic transient depression; $(n=7)$. Analog traces represent typical field EPSPs 30 min before the normal time point " 0 ," i.e., the time point where SLFS or WLFS was applied (dashed line); $30 \mathrm{~min}$ (solid line) and $6 \mathrm{~h}$ (dotted line) after LFS of input $p S$ or $d S$. The analog traces for the corresponding control inputs were recorded at the same time points. Calibration bar for all analog traces: $3 \mathrm{mV} / 5 \mathrm{~ms}$. Arrows indicate the time point of stimulation of synaptic input $\mathrm{pS}$ or $\mathrm{dS}$ (SLFS is symbolized as dashed arrow, WLFS by gray, dashed arrow). The neuronal schemes presented in each data panel should emphasize the used stimulation protocol in the corresponding experiment: $\mathrm{pS}$ or $\mathrm{dS}$.

different synaptic inputs at the apical dendrite can functionally and associatively interact in distinct circumstances, we have also investigated whether processes of "synaptic tagging" and "synaptic cross-tagging," i.e., the late-associative interaction of LTD and LTP at different synaptic inputs, can occur (Sajikumar and Frey, 2004; Sajikumar et al., 2005b).

\section{Materials and Methods}

All experiments were performed in accordance with the European Community Council Directive of 24 November 1986 (86/609/EEC). We also certify that formal approval to conduct the experiments has been obtained from the animal subject review board of our institution/ local government, which can be provided upon request. All efforts were made to minimize the number of animals used and their suffering.

Slice preparation and electrophysiology. One hundred and one transverse hippocampal slices (400- $\mu \mathrm{m}$-thick), prepared from 101 male Wistar rats (7-week-old), were used as described previously (Frey et al., 1988; Sajikumar et al., 2005a). In all experiments, two monopolar lacquer-coated, stainless-steel electrodes (all electrodes were obtained from A-M Systems) were positioned within the stratum radiatum of the CA1 for stimulating two independent synaptic inputs, $\mathrm{pS}$ (proximal part) or dS (distal part; Fig. 1A). The pathway independency was assessed as described previously (Dunwiddie and Lynch, 1978; Frey et al., 1988). Furthermore, the absence of paired-pulse facilitation between the two inputs suggested the stimulation of independent pathways [interpulse interval: $50 \mathrm{~ms}$, stimulus intensity as test recording; this particular stimulation resulted in a facilitation of the second potential if delivered via $\mathrm{pS}$ or $\mathrm{dS}$, however, if the two stimuli were alternatively applied to $\mathrm{pS}$ or $\mathrm{dS}$ (or vice versa), no facilitation in the second potential was detected]. For recording the field EPSP (measured as its slope function at its steepest part) and the population spike amplitude (PS), two electrodes were placed in the CA1 apical dendritic and cell body layer, respectively, of a single neuronal population to investigate plasticity in the apical dendritic branches. Recorded signals were analyzed on-line as described previously; slices were preincubated for at least $4 \mathrm{~h}$, which is critical for reliable long-term recordings of LTD (for more details, see Sajikumar et al., 2005a). Following the preincubation period, the test stimulation strength was determined for each input to elicit a PS of $40 \%$ of its maximal amplitude for all control and LTD inputs and $25 \%$ for LTP inputs.

Late-LTD was induced using a strong, lowfrequency stimulation (SLFS) protocol of 900 bursts (a single burst consisted of three biphasic, constant current stimuli at $f=20 \mathrm{~Hz}$, interburst interval: $1 \mathrm{~s}$; stimulus duration: 0.2 $\mathrm{ms} /$ half-wave; total number of stimuli: 2700). This stimulation pattern produced a stable late-LTD in vitro for $6 \mathrm{~h}$, the end of the experiments. In experiments in which a weaker induction of LTD was investigated, a weak, lowfrequency stimulation protocol (WLFS) was used consisting of 900 pulses at a frequency of 1 $\mathrm{Hz}$, impulse duration of $0.2 \mathrm{~ms} /$ half-wave, with 900 total biphasic stimuli. Late-LTP was induced using three stimulus trains of 100 pulses per train ["strong" tetanus (STET), $f=100 \mathrm{~Hz}$; impulse duration: $0.2 \mathrm{~ms} /$ polarity; intertrain interval: $10 \mathrm{~min}$ ]. In experiments with induction of early-LTP, a weak tetanization protocol (WTET) consisting of one train (21 biphasic constant-current pulses; $f=100 \mathrm{~Hz}$; pulse width duration $0.2 \mathrm{~ms} /$ polar- 
ity; TET-stimulus intensity: 25\% of maximal field EPSP) was used. Four biphasic constant-current pulses ( $f=0.2 \mathrm{~Hz}$, impulse duration: $0.1 \mathrm{~ms}$ per polarity) were used for baseline recordings (every $15 \mathrm{~min}$ before tetanization or LFS) and testing at 1,3,5,11, 15, 21, 25, and 30 min after tetanus or after LFS and then every 15 min up to $6 \mathrm{~h}$, i.e., the end of the experiment. The PS and the slope of the field EPSP were monitored on-line. For clarity, the field EPSP data are only shown because the two recorded parameters revealed similar time courses in all experiments.

Pharmacological substances. The myristoylated pseudosubstrate peptide, myr- $\zeta$ inhibitory peptide (myr-ZIP; Biosource) was dissolved in distilled water and maintained as a stock solution $(10 \mathrm{mM})$ and stored at $-20^{\circ} \mathrm{C}$. The required volume containing the final concentration of $1 \mu \mathrm{M}$ (Sajikumar et al., 2007) was dissolved in ACSF immediately before bath application.

Statistical analysis of electrophysiological data. Averaged values of the slope function of the field EPSP (expressed as their percentage change to baseline recordings within the $1 \mathrm{~h}$ baseline recording) per time point were analyzed using the nonparametric Wilcoxon signed-rank test when compared within one group, or the Mann-Whitney $U$ test when data were compared between groups ( $p$ values $<0.05$ were considered as statistically significantly different).

\section{Results}

SLFS and WLFS applied to different apical dendritic sites

In a first set of experiments SLFS was applied to the proximal part of the apical branch of CA1 dendrites pS. Only a short-term depression (STD) was observed in the pS-input (Fig. $1 B$, filled circles). Interestingly, control stimulation to the distal input dS was also transiently influenced by the SLFS to pS (Fig. $1 B$, open circles). Both inputs expressed a short-term depression, whereas the depression observed in $\mathrm{dS}$ was of a heterosynaptic nature. The depression in pS (Fig. $1 B$, filled circles) was not statistically significantly different from potentials of the control dS pathway (Fig. $1 B$, open circles; $U$ test, $p>0.05$ ). The short-term potentiation was statistically different only for the first $21 \mathrm{~min}$ after the end of SLFS in pS, whereas in dS it was for $30 \mathrm{~min}$ when compared with its own baseline values before stimulation (Wilcoxon $t$ test, $p<0.05$ ). The potentials returned to baseline levels, remaining there up to the sixth hour after SLFS to pS. In the next series of experiments (Fig. 1C) we investigated whether late-LTD can be induced in the distal part of the apical dendrites dS. SLFS of synaptic input dS (Fig. 1C, filled circles) resulted in a persistent, statistically significant L-LTD with a duration of $6 \mathrm{~h}$, when compared with the values in the control-stimulated pathway pS (Fig. $1 C$, open circles; $U$ test, $p<0.05$ ) or its own baseline values before stimulation (Wilcoxon test, $p<0.05$ ). The control input, $\mathrm{pS}$, remained stable at baseline levels for the entire experimental session with the exception of a short-term, heterosynaptic depression during the first $11 \mathrm{~min}$ after the end of SLFS to dS (Fig. 1C, open circles; Wilcoxon, $p<0.05$ ).

Subsequently, we were interested to see what effect a WLFS, normally resulting in E-LTD, would have at the different synaptic inputs $\mathrm{pS}$ and $\mathrm{dS}$ of the apical CA1 dendrite. Application of WLFS in $\mathrm{pS}$ (Fig. $1 D$, filled circles) resulted only in a slight STD, which was statistically significantly different from the potentials recorded from the control pathway dS (Fig. $1 D$, open circles) within 45 min after the end of WLFS ( $U$ test, $p<0.05$ ) or significantly different from its own baseline values before WLFS for $21 \mathrm{~min}$ after the end of WLFS application (Wilcoxon, $p<0.05$ ). The control input $\mathrm{dS}$, without WLFS, remained stable at baseline levels for the entire experimental session after $20 \mathrm{~min}$ of SLFS induction (Fig. 1D, open circles). Thereafter, potentials in both inputs returned to baseline levels, where they remained until the end of the experiment.

WLFS to dS (Fig. 1E, filled circles) resulted in a transient E-LTD with a duration of $165 \mathrm{~min}$ when compared with its baseline values before WLFS. In the control pathway $\mathrm{pS}$, a heterosyn- aptic STD was observed which was statistically significantly different for 15 min after the end of WLFS to dS (Wilcoxon test, $p<0.05)$. After reaching the baseline, the potentials in both inputs then remained stable up to the end of the experiment.

\section{SLFS, WTET, and STET and cross-tagging between $\mathrm{pS}$ and $\mathrm{dS}$}

We were now interested to investigate whether processes of synaptic tagging and cross-tagging could be observed in the $\mathrm{pS}$ and dS. Thus, the first set of experiments was undertaken just to confirm previous results from our laboratory (Sajikumar and Frey, 2004): a SLFS was applied to the dS (Fig. 2A, open circles) followed by a WTET to pS $45 \mathrm{~min}$ later (Fig. $2 A$, filled circles). Late-LTD was induced in dS by SLFS, which was maintained up to the end of the experiment. WTET to pS, which normally results in E-LTP (for comparison see Sajikumar and Frey, 2004) was paradoxically transformed into L-LTP if preceded by SLFS to dS. According to the synaptic tagging hypothesis (Frey and Morris, 1997; Sajikumar and Frey, 2004), SLFS sets its transient LTD-tags and activates the synthesis of plasticity-related proteins (PRPs) (Sajikumar et al., 2007). If within an effective time window (Frey and Morris, 1998b; Frey and Frey, 2008) an independent synaptic input $\mathrm{pS}$ is activated by a WTET, which sets its synapse-specific LTP-tags but is unable to induce its own synthesis of PRPs, then this input $\mathrm{pS}$ could benefit from the PRPs synthesized by the SLFS in dS. The LTP-pS-tag complex could then capture the PRPs and thus transforming its normally E-LTP into L-LTP. As shown in Figure $2 A$ the normally transient E-LTP in pS was indeed transformed into L-LTP by the prior induction of L-LTD in dS. Furthermore, we had earlier shown that the atypical isoform of protein kinase (PK) C-PKMzeta-is a LTP-specific PRP in apical CA1 dendrites (Sajikumar et al., 2005b). Thus, next we investigated whether PKMzeta inhibition by myr-ZIP could affect the maintenance of L-LTP induced via cross-tagging. Therefore, we repeated the experiment as in Figure $2 A$, but now, myr-ZIP was applied $30 \mathrm{~min}$ after WTET to pS. myr-ZIP remained in the bath for $4 \mathrm{~h}$. Interestingly, L-LTD in dS was unaffected (open circles), whereas LTP in pS (filled circles) now also expressed only E- but not L-LTP. Thus, in contrast to cross-tagging as shown in Figure $2 A$, myr-ZIP prevented the transformation of E-LTP in to L-LTP in $\mathrm{pS}$ by the prior L-LTD induction in $\mathrm{dS}$. The potentiation in $\mathrm{pS}$ was only detected for up to $2 \mathrm{~h}$ and 45 min after WTET to $\mathrm{pS}$ (Wilcoxon, $p<0.05$ ). The initial potentiation level in both series was not statistically significantly different for up to $45 \mathrm{~min}$ after tetanization ( $p>0.05, U$ test). Interestingly, we could not observe a further decline of potentials below baseline after LTP induction and the application of myr-ZIP within the here measured first 5-6 h. This is in line with results described by others (Serrano et al., 2005). However, we cannot exclude a decline below baseline at later time points as it was the case in our earlier experiments (Sajikumar et al., 2005b).

The next set of experiments shown in Figure $2 C$ was similar to Figure $2 A$, but now we changed the order of stimulation at the corresponding inputs, i.e., SLFS was now applied to pS and WTET to dS. As shown in Figure $1 B$, SLFS to pS did not result in L-LTD. However, the question arose whether, regardless of that fact, the SLFS was still able to induce PRPs relevant for LTP. SLFS to $\mathrm{pS}$ resulted in STD with a duration of $21 \mathrm{~min}$ after the end of SLFS-application. Then, the potentials returned to baseline where they remained until the end of the experiment (Fig. 2C, filled circles; Wilcoxon test), similar to that shown in Figure $1 B$; and regardless of further WTET to dS. SLFS to pS resulted also in a heterosynaptic transient depression in $\mathrm{dS}$ which was back to baseline values $21 \mathrm{~min}$ after the end of SLFS to pS (Fig. 2C, open 
A

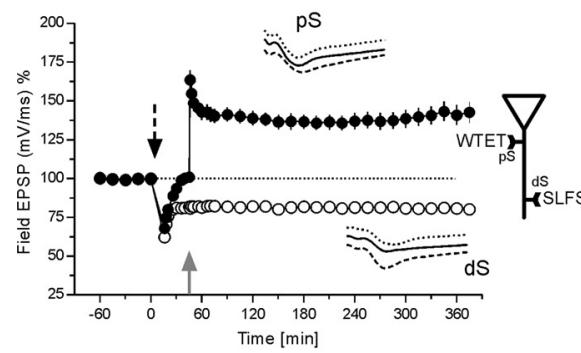

C

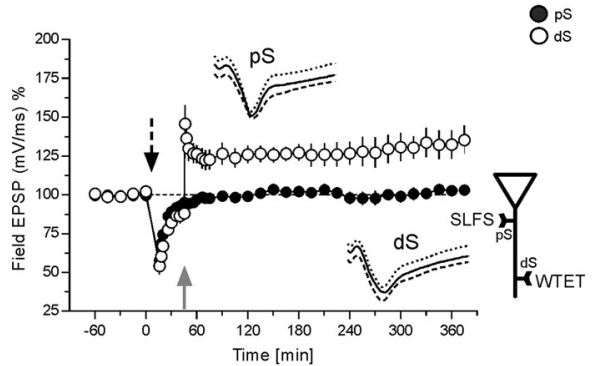

E

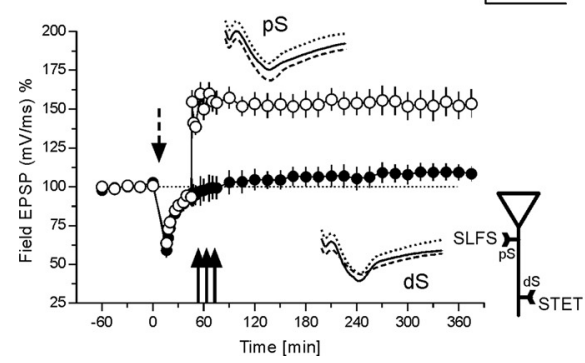

B

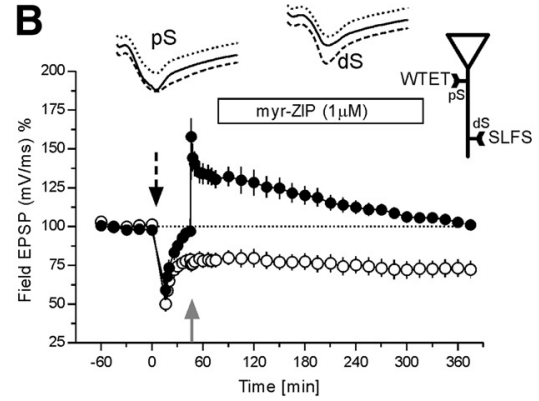

D

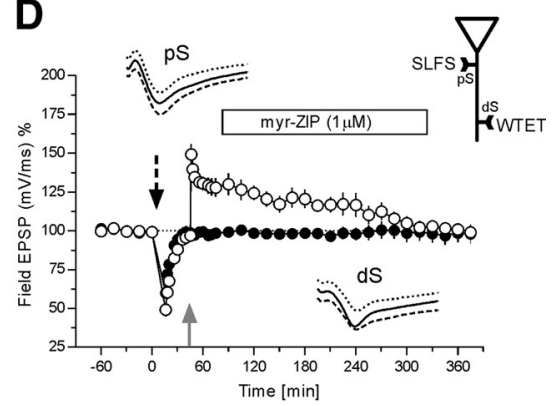

$\mathbf{F}$

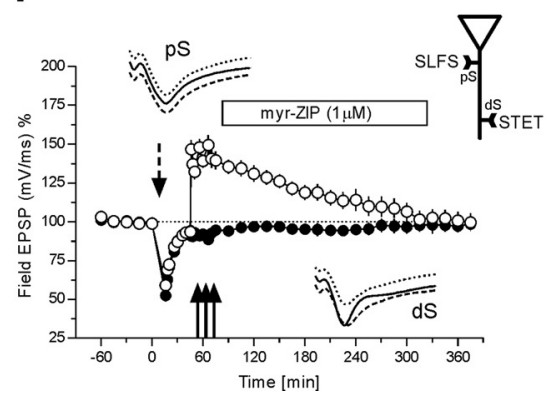

Figure 2. Associative properties of LTD forms induced at different locations of apical CA1 neurons. The time courses of field EPSP recordings are provided. $\boldsymbol{A}$ represents a control experiment verifying associative properties of synaptic cross-tagging (see Results for more detail). The time course of the slope of the field EPSP after SLFS dS (open circles) is presented. Thirty min after the end of SLFS to dS or 45 min after its beginning, a WTET to $\mathrm{pS}$ was applied (filled circles). The normally E-LTP in pS was transformed in to L-LTP by the prior induction of late-LTD in dS; $(n=14)$. $B$, The time course of a cross-tagging experiment similar to that in $\boldsymbol{A}$ is presented, however, with the application of myr-ZIP (1 $\mu \mathrm{M})$ after WTET to pS (filled circles, box represents drug application). Myr-ZIP was applied 30 min after WTET of $p S$ for a duration of $4 \mathrm{~h} ;(n=9)$. Synaptic cross-tagging in $p S$ was blocked by myr-ZIP, i.e., the transformation of E-LTP into L-LTP was prevented. There was no change in maintenance of late-LTD at the synaptic input dS (open circles). $\boldsymbol{C}$, An experiment similar to that in $\boldsymbol{A}$; however, here, instead of WTET to pS, SLFS was applied to $\mathrm{pS}$ (filled circles) and instead of SLFS to dS, a WTET was applied to DS (open circles; $n=9$ ). E-LTP induced in $\mathrm{pS}$ was still transformed into L-LTP by the prior application of SLFS to pS. D, Protocol similar to that in $\boldsymbol{C}$, but now myr-ZIP was applied 30 min after WTET to dS which prevented the transformation of E- into L-LTP in dS (open circles; $n=7$ ). $\boldsymbol{E}$, A procedure similar to that in $\boldsymbol{C}$, with the exception that instead of a WTET, a STET was applied to dS (open circles). The time course of STD induced a priori by SLFS in pS did not change (filled circles; $n=9$ ). $\boldsymbol{F}$, An experiment similar to that in $\boldsymbol{E}$, with the exception that myr-ZIP was applied 30 min after STET application to dS (open circles). Late-LTP in dS was thus blocked; however, no change in the time course of STD was observed in pS, which was previously induced by the application of SLFS (filled circles; $n=10$ ). Analog examples as in Figure 1, with the exception that solid line represents potentials recorded $30 \mathrm{~min}$ after the second stimulation event. Gray solid arrow represents the time point of WTET; the triplet of black solid arrows indicates the time points of STET. The bars in $\boldsymbol{B}, \boldsymbol{D}$, and $\boldsymbol{E}$ represent the time period in which myr-ZIP was applied. Other abbreviations and symbols are as in Figure 1.

circles; Wilcoxon test). WTET dS, 45 min after the start of SLFS to pS, however, revealed the paradoxical induction of L-LTP in dS, suggesting that LTP-tags set by WTET to dS could capture PRPs, the synthesis of which must have been induced by the SLFS to pS. To verify this assumption, we repeated the same experiment, but now with application of myr-ZIP (as in Fig. $2 B$ ), assuming the SLFS to $\mathrm{pS}$ induced the synthesis of the LTP-specific PRP, PKMzeta, which was then bound to LTP-tags in dS, thus transforming E-LTP into L-LTP. As shown in Figure $2 D$, application of myr-ZIP indeed blocked the transformation of E-LTP into
L-LTD [LTP returned to baseline $3 \mathrm{~h}$ and 15 min after WTET to dS; Fig. 2D, open circles; Wilcoxon $t$ test; the initial potentiation level in both series was not statistically significantly different for up to 45 min after tetanization ( $p>0.05, U$ test $)]$, supporting our assumption that SLFS to pS induced the synthesis of LTP-specific PRPs, including PKMzeta, without being able to induce a longer-lasting LTD in its stimulated input by itself.

Next, we hypothesized that, although SLFS to $\mathrm{pS}$ was unable to induce any longer-lasting depressions there (Fig. 1), it could be possible that the SLFS could still set LTD-specific tags at least. If so, then a strong event, such as STET to another independent synaptic input (here dS), should result in L-LTD in pS by means of cross-tagging mechanisms, if STET is applied within an effective time window. The assumed LTD-tags at $\mathrm{pS}$ would then be able to capture PRPs, the synthesis of which was induced by the STET to dS (Sajikumar and Frey, 2004). Figure $2 E$ shows the result: in contrast to our hypothesis, STD in pS induced by SLFS (filled circles) was not affected even if STET was applied to dS (Fig. 2E, open circles) within an effective time window (45 min after the start of SLFS to pS). Only STD in pS was detected, similar to what was shown in the earlier experiments. The last series of experiments was just undertaken to show that strong events (e.g., SLFS and STET to two separate inputs) still remain specific; i.e., L-LTP normally induced by STET to dS was prevented if myrZIP was applied $30 \mathrm{~min}$ after STET to dS [Fig. $2 F$, open circles; the potentiation was statistically significantly different from its own baseline values only for $3 \mathrm{~h}$ and $15 \mathrm{~min}$ after STET, Wilcoxon- $t$ test; again, the initial potentiation level in both series was not statistically significant after tetanization and before drug application $(p>0.05, U$ test $)]$.

\section{Discussion}

We were interested to study whether LTD induction, its maintenance, and associative properties could differ at excitatory synaptic inputs located proximally or distally on the apical dendritic tree of CA1 pyramidal neurons. Surprisingly, we did not observe any longer-lasting forms of LTD at the proximal region (Fig. $1 B, D$ ). This result is in line with data reported from others (Paulsen et al., 1993). However, at the distal region we were able to induce reliably L-LTD and E-LTD (Fig. 1C,E). These results are in accordance with data obtained in pyramidal neurons from layer $2 / 3$ in rat cortical slices using whole-cell recording techniques: LTD was absent at proximal but was still observed at distal synapses (Froemke et al., 2005). Further, studies by others, as well as our own, have shown that different forms of LTD 
can be induced in the apical dendrites of CA1 pyramidal neurons under distinct circumstances (Kemp et al., 2000; ManahanVaughan, 2000; Manahan-Vaughan et al., 2000; Sajikumar and Frey, 2003, 2004; Malenka and Bear, 2004; Pöschel and Manahan-Vaughan, 2007). Here, we clearly demonstrate that longer-lasting forms of LTD can only be induced in the distal part of the apical dendrites of CA1 pyramidal neurons. Proximal inputs showed only transient forms of depression, either homosynaptically or heterosynaptically induced, however, always lasting only a few minutes. With respect to the general requirements to induce lasting forms of LTD in the hippocampal CA1, some groups have suggested that the efficacy to induce LTD drops with age $>40$ d in rats, or alternate induction protocols are required to induce LTD, such as paired-pulse combined with LFS (Bashir and Collingridge, 1994; Kemp and Bashir, 1999; Kemp et al., 2000). Although we agree also that the role of age and the stimulus protocol can influence LTD in the CA1 region (Frey and Morris, 1998a; Sajikumar et al., 2005a), we could clearly show that the determining factor to induce LTD is, in addition to the age and stimulus protocol, specifically, the location of synapses in the apical dendritic branch, at least in young adult animals at an age of 7 weeks. We could further show that distally located synaptic inputs in the apical dendrite of CA1 neurons can express different forms of LTD and transient heterosynaptic events as well as E-LTD and L-LTD.

With respect to its late-associative interactions in apical dendrites, we have also investigated processes of synaptic tagging and cross-tagging. The concept of synaptic tagging can explain synaptic input specificity during long-lasting plasticity events. Specific LFS or high-frequency stimulation can activate and set process-specific, i.e., LTD- or LTP-specific, tags at the activated input. Heterosynaptic inputs, including neuromodulatory inputs, are required to induce the synthesis of PRPs, which bind to the glutamatergic, process-specific "tagged" synapses and consequently expressing L-LTD or L-LTP (Frey and Morris, 1997; Sajikumar and Frey, 2004). Field stimulation within the stratum radiatum always results also in the activation of other than glutamatergic synapses. Thus, functional synapses from extrahippocampal neuromodulatory inputs are always activated as well as (Frey et al., 1990; Frey and Morris, 1998a). We have previously shown that either SLFS or STET in the stratum radiatum consequently activates glutamatergic as well as dopaminergic receptors, the function of which is required specifically to induce the synthesis of PRPs (Frey et al., 1993; Frey and Morris, 1998a; Sajikumar and Frey, 2004; Navakkode et al., 2007). In addition to synaptic tagging, the functional, spaced-associative interaction between LTP and LTD can be studied with the "cross-tagging" model (Sajikumar and Frey, 2004; Sajikumar et al., 2007; Frey and Frey, 2008). In that case, LTD- or LTP-specific tags can benefit from PRPs, regardless of whether the synthesis was induced by LTP, LTD, neuromodulatory, or other processes. If related PRPs are provided together with tag activity within a time window, an even normally transient, opposite plasticity form can be transformed into a long-lasting one. In other words, if E-LTP is induced in a synaptic input with its LTP-tags set, it just requires the availability of PRPs regardless from which other process they were induced. If through an independent synaptic input in the same functional dendritic compartment L-LTD is induced, for which it is known that it activates the synthesis of a pool of PRPs, then the LTP-tags can capture the "strange" PRPs and thus transform E-LTP into L-LTP, by means of the result of L-LTD induction in an independent input (Frey and Frey, 2008). We have shown, here, first that distally located synaptic inputs were able to activate the synthesis of PRPs which transformed weakly potentiated proximal synaptic inputs into persistently potentiated synapses (Fig. 2A). Furthermore, application of myr-ZIP prevented the transformation of E-LTP into its late form at proximal inputs while not affecting the maintenance of L-LTD in distal locations (Fig. $2 \mathrm{~B}$ ). Our previous results indicated that PKMzeta is a LTP-specific PRP; thus only L-LTP but not L-LTD was affected (Sajikumar et al., 2007). Interestingly, when the locations were reversed in the above set of experiments, i.e., SLFS was applied to proximal inputs whereas WTET to distal inputs (Fig. 2C), E-LTP at the distal inputs was still reinforced into a L-LTP also if proximally applied SLFS did not result in a long-lasting event in that location. This result suggests that SLFS to proximal inputs can induce the synthesis of PRPs specifically at the proximal input including PKMzeta, without setting its LTDspecific tags [ the latter is supported by the fact that STET to distal inputs could not interact with SLFS at proximal inputs (Fig. $2 E, F)]$. That would result only in short-term LTD at proximal synapses but would explain the late-associative effects distally. The ability of proximal synapses to synthesize LTP-specific PRPs when subjected to SLFS is quite intriguing while the same synapses are unable to maintain E-LTD or L-LTD under our conditions. On the other hand, SLFS at proximal sites, if strong enough, may unspecifically cause the release of dopamine which, by itself, in a synergistic interaction with STET at distal synapses, could induce the required synthesis of PRPs. Alternatively, SLFS in the proximal location is only able to activate the synthesis of LTP-specific PRPs but not those required for LTD; or the used low-frequency stimulation protocols were suboptimal to set LTD-specific tags and PRPs at the proximal input. Future studies must shed light into these questions.

Why does SLFS not result in LTD at proximal sites? Activation of L-type voltage-dependent calcium channels (VDCC) seems to be essential to induce LTD in some preparations. Thus, one could hypothesize that plasticity forms at proximally located inputs could be characterized by VDCC activation, resulting in more $\mathrm{Ca}^{2+}$ influx, which shifts the balance from depression to LTP. Thus competing mechanisms at this part of the dendrite could limit the maintenance of LTD. Locally generated and spatially restricted regenerative potentials (dendritic spikes) could also contribute to the postsynaptic depolarization and enhanced calcium entry at these sites shifting the balance to LTP instead of LTD (Golding et al., 2002). Another explanation for the absence of lasting LTD forms at proximal sites could be due to a more effective GABAergic innervation in the proximal part of the apical dendrites when compared with the distal region (Megías et al., 2001), i.e., LFS does not result in the needed activation of postsynaptic events in the pyramidal cell. Only further studies will clarify why SLFS in proximal dendrites does not result in longer-lasting forms of LTD.

From a more conceptual way of thinking, one could also search for a possible explanation on the level of network formation. Unfortunately, less is known about how, where, and with which structures the different loci of the apical dendrites form functional networks and why it might be useful for the system to express at some places LTP and LTD, at other loci only one of the forms, or eventually no plasticity event at all.

\section{References}

Barry MF, Vickery RM, Bolsover SR, Bindman LJ (1996) Intracellular studies of heterosynaptic long-term depression (LTD) in CA1 of hippocampal slices. Hippocampus 6:3-8.

Bashir ZI, Collingridge GL (1994) An investigation of depotentiation of 
long-term potentiation in the CA1 region of the hippocampus. Exp Brain Res 100:437-443.

Dunwiddie T, Lynch G (1978) Long-term potentiation and depression of synaptic responses in the rat hippocampus: localization and frequency dependency. J Physiol 276:353-367.

Frey S, Frey JU (2008) 'Synaptic tagging' and 'cross-tagging' and related associative reinforcement processes of functional plasticity as the cellular basis for memory formation. Prog Brain Res 169:117-143.

Frey U, Morris RG (1997) Synaptic tagging and long-term potentiation. Nature 385:533-536.

Frey U, Morris RG (1998a) Synaptic tagging: implications for late maintenance of hippocampal long-term potentiation. Trends Neurosci 21:181188.

Frey U, Morris RG (1998b) Weak before strong: dissociating synaptic tagging and plasticity-factor accounts of late-LTP. Neuropharmacology 37:545-552.

Frey U, Krug M, Reymann KG, Matthies H (1988) Anisomycin, an inhibitor of protein synthesis, blocks late phases of LTP phenomena in the hippocampal CA1 region in vitro. Brain Res 452:57-65.

Frey U, Schroeder H, Matthies H (1990) Dopaminergic antagonists prevent long-term maintenance of posttetanic LTP in the CA1 region of rat hippocampal slices. Brain Res 522:69-75.

Frey U, Huang YY, Kandel ER (1993) Effects of cAMP simulate a late stage of LTP in hippocampal CA1 neurons. Science 260:1661-1664.

Froemke RC, Poo MM, Dan Y (2005) Spike-timing-dependent synaptic plasticity depends on dendritic location. Nature 434:221-225.

Golding NL, Staff NP, Spruston N (2002) Dendritic spikes as a mechanism for cooperative long-term potentiation. Nature 418:326-331.

Kemp N, Bashir ZI (1999) Induction of LTD in the adult hippocampus by the synaptic activation of AMPA/kainate and metabotropic glutamate receptors. Neuropharmacology 38:495-504.

Kemp N, McQueen J, Faulkes S, Bashir ZI (2000) Different forms of LTD in the CA1 region of the hippocampus: role of age and stimulus protocol. Eur J Neurosci 12:360-366.

Malenka RC, Bear MF (2004) LTP and LTD: an embarrassment of riches. Neuron 44:5-21.

Manahan-Vaughan D (2000) Long-term depression in freely moving rats is dependent upon strain variation, induction protocol and behavioral state. Cereb Cortex 10:482-487.

Manahan-Vaughan D, Kulla A, Frey JU (2000) Requirement of translation but not transcription for the maintenance of long-term depression in the CA1 region of freely moving rats. J Neurosci 20:8572-8576.
Massey PV, Bashir ZI (2007) Long-term depression: multiple forms and implications for brain function. Trends Neurosci 30:176-184.

Megías M, Emri Z, Freund TF, Gulyás AI (2001) Total number and distribution of inhibitory and excitatory synapses on hippocampal CA1 pyramidal cells. Neuroscience 102:527-540.

Mizuno T, Kanazawa I, Sakurai M (2001) Differential induction of LTP and LTD is not determined solely by instantaneous calcium concentration: an essential involvement of a temporal factor. Eur J Neurosci 14:701-708.

Mulkey RM, Endo S, Shenolikar S, Malenka RC (1994) Involvement of a calcineurin/inhibitor-1 phosphatase cascade in hippocampal long-term depression. Nature 369:486-488.

Navakkode S, Sajikumar S, Frey JU (2007) Synergistic requirements for the induction of dopaminergic D1/D5-receptor-mediated LTP in hippocampal slices of rat CA1 in vitro. Neuropharmacology 52:1547-1554.

Paulsen O, Li YG, Hvalby O, Anderson P, Bliss TV (1993) Failure to induce long-term depression by an anti-correlation procedure in area CA1 of the rat hippocampal slice. Eur J Neurosci 5:1241-1246.

Pöschel B, Manahan-Vaughan D (2007) Persistent (>24 h) long-term depression in the dentate gyrus of freely moving rats is not dependent on activation of NMDA receptors, L-type voltage-gated calcium channels or protein synthesis. Neuropharmacology 52:46-54.

Sajikumar S, Frey JU (2003) Anisomycin inhibits the late maintenance of long-term depression in rat hippocampal slices in vitro. Neurosci Lett 338:147-150.

Sajikumar S, Frey JU (2004) Late-associativity, synaptic tagging, and the role of dopamine during LTP and LTD. Neurobiol Learn Mem 82:12-25.

Sajikumar S, Navakkode S, Frey JU (2005a) Protein synthesis-dependent long-term functional plasticity: methods and techniques. Curr Opin Neurobiol 15:607-613.

Sajikumar S, Navakkode S, Sacktor TC, Frey JU (2005b) Synaptic tagging and cross-tagging: the role of protein kinase Mzeta in maintaining longterm potentiation but not long-term depression. J Neurosci 25:57505756.

Sajikumar S, Navakkode S, Frey JU (2007) Identification of compartmentand process-specific molecules required for "synaptic tagging" during long-term potentiation and long-term depression in hippocampal CA1. J Neurosci 27:5068-5080.

Serrano P, Yao Y, Sacktor TC (2005) Persistent phosphorylation by protein kinase Mzeta maintains late-phase long-term potentiation. J Neurosci 25:1979-1984. 